\title{
The management of upper gastrointestinal haemorrhage in a tropical country
}

\author{
T. H. S. G. RAO, G. K. PANDE, P. SAHNI \& \\ S. NUNDY
}

Department of Gastrointestinal Surgery and Liver Transplantation, All India Institute of Medical Sciences, New Delhi 110029, India

\section{INTRODUCTION}

The management of a patient with haematemesis and melaena can be a formidable task for a treating doctor especially if he is confronted with the problem for the first time. This difficulty is compounded in the tropics because very little has been written about the problems of treating such patients in these countries (Tandon, 1978). The causes of haemorrhage are different, (Wicks et al., 1975; Morgan et al., 1977; Anand et al., 1983) sophisticated instruments for diagnosis are not available, there is a shortage of blood for transfusion and skilled and experienced surgeons are rare. This article aims to place in perspective the problem of upper gastrointestinal (GI) haemorrhage in the tropics particularly in India and discusses appropriate methods of management.

\section{AETIOLOGY}

The three main causes of upper GI bleeding all over the world are peptic ulcer, oesophageal varices and erosive mucosal disease (EMD) but the relative frequency of these conditions vary in the different countries (Table 1). Thus in the U.S.A. patients with bleeding peptic ulcers constitute about $50 \%$ of admissions with upper GI haemorrhage, patients with portal hypertension constitute about $15 \%$ and those with erosive mucosal diseases of the stomach and duodenum $28 \%$. In the U.K. on the other hand, although approximately the same proportions of patients bleed from peptic ulcer and erosions, variceal bleeding is much less common constituting only $2 \%$ of all episodes.

Correspondence: $\operatorname{Dr}$ S. Nundy, Additional Professor and Head, Department of G. I. Surgery and Liver Transplantation, All India Institute of Medical Sciences, Nerw Delhi 110029, India. 
Table 1. The causes of upper gastrointestinal haemorrhage in tropical and Western countries.

\begin{tabular}{|c|c|c|c|c|c|}
\hline \multicolumn{2}{|c|}{$\begin{array}{c}\text { Country } \\
\text { (Ref) }\end{array}$} & \multirow{2}{*}{$\begin{array}{c}\begin{array}{c}\text { Varices } \\
(\%)\end{array} \\
45\end{array}$} & \multirow{2}{*}{$\begin{array}{c}\begin{array}{c}\text { Ulcer } \\
\%\end{array} \\
30\end{array}$} & \multirow{2}{*}{$\begin{array}{c}\begin{array}{c}\text { EMD } \\
\%\end{array} \\
9\end{array}$} & \multirow{2}{*}{$\begin{array}{c}\begin{array}{c}\text { Others } \\
\%\end{array} \\
16\end{array}$} \\
\hline India & (Anand et al., 1983) & & & & \\
\hline U.S.A. & (Yajko et al., 1975) & 15 & 51 & 28 & 4 \\
\hline U.K. & (Allan \& Dykes, 1976) & 2 & 49 & 17 & 32 \\
\hline
\end{tabular}

EMD - Erosive mucosal disease

Ulcer - Gastric and duodenal ulcer

In India and many other tropical countries the largest group of patients have $\overrightarrow{\mathscr{\omega}}$ variceal bleeding and constitute $45 \%$ of the total, $30 \%$ have a bleeding peptic ulcer $\frac{\Phi}{3}$ and about $10 \%$ bleed from erosive mucosal disease. The remaining patients in both developed and developing countries bleed from diverse causes including $\omega$ oesophagitis, carcinoma of the oesophagus and stomach, and gastric leiomyomas. $\vec{\circ}$ These conditions are rare and need specific approaches to management and weo will not discuss them further. In up to $10 \%$ of patients no cause of bleeding may $\overrightarrow{0}$ be found.

\section{INCIDENCE AND PATIENT CHARACTERISTICS}

Most large hospitals in the world admit between 100 and 200 cases with upper bleeding every year (Schiller et al., 1970; Forrest \& Finlayson, 1974) and in our own 1000 bedded general hospital we admit from 150-200 annually. However, our patients differ from western patients. In Western countries upper GI haemorrhage $\bar{D}$ occurs in patients between 50 and 80 years of age (mean age 55 years), in tropical $\overrightarrow{\vec{P}}$ countries the patients are younger (mean age 41 years). There is also a slightly 3 greater preponderance of males over females (3:1) in tropical countries than in the West (2:1).

\section{MANAGEMENT}

In tropical countries, sophisticated medical facilities are scarce except for a few $\stackrel{?}{?}$ specialized centres. Most hospitals do not have blood banks, fibreoptic endoscopes $\frac{\vec{T}}{\square}$ or equipment for angiography. However, we believe that sophisticated methods of diagnosis and treatment are not usually necessary and based on our experience. of 1480 patients with upper GI haemorrhage whom we have looked after over 0 the last 14 years (1976-1989), we have evolved a simple stepwise approach to $\omega$ their management.

The first step is to resuscitate the patient, the second to make a tentative $\frac{0}{\Phi}$ diagnosis, the third to allocate the patient to a low-risk group comprising patients $\stackrel{f}{?}$ 
who can be managed fairly adequately at home or a high-risk group comprising patients who need hospital treatment (details of which constitute the fourth step).

\section{Step I: Resuscitation}

If the patient with upper GI haemorrhage has systolic blood pressure below $100 \mathrm{mmHg}$ and a pulse rate greater than $100 \mathrm{~min}^{-1}$ the first action should be to restore his blood volume. This is done by starting two good and reliable intravenous lines using wide bore needles inserted into each antecubital vein at the elbow and running in four bottles of $5 \%$ dextrose or normal saline as quickly as possible. Under- rather than over-transfusion is the more common error in these patients and whilst it is important to look for signs of congestive heart failure it is more important to restore the blood pressure to normal levels. If colloids such as plasma, Dextran (Pharmacia, Sweden) or Haemaccel (Hoechst, Germany) are available they should be given, remembering they are more viscous and less easily infused than crystalloids. They also interfere with blood crossmatching and a $10 \mathrm{ml}$ sample of blood should be taken for blood grouping and crossmatching.

After restoring the systolic blood pressure to above $100 \mathrm{mmHg}$ it is necessary to pass a large $(16 \mathrm{~F})$ Levine tube into the stomach, aspirate its contents and wash it with ice-cold saline and antacids every $2 \mathrm{~h}$. If bleeding persists, we instil a weak solution of noradrenaline $(8 \mathrm{mg}$ in $100 \mathrm{ml}$ saline) into the stomach every $30 \mathrm{~min}$ for $4 \mathrm{~h}$. In a patient who has had hypotension for longer than $1 \mathrm{~h}$ a urinary catheter should be inserted and the hourly urine output maintained at above $30 \mathrm{ml}$. This simple measure is a good way of assessing the adequacy of tissue perfusion and of fluid replacement. Insertion of central venous pressure lines are not very useful in tropical countries because our nurses are often hesitant to manipulate the fluid column system and in addition the lines are a potent source of serious infection. The hourly urine output together with visual estimation of the jugular venous pressure and auscultation of the lung bases for crepitations are usually adequate to assess the restoration of intravenous fluid volume.

Once the patient is haemodynamically stable the clinician can proceed to the next step which is to make a diagnosis.

\section{Step II: Diagnosis}

An easy guide to diagnosis is the presence of splenomegaly. If the spleen is palpable the patient is likely to be bleeding from oesophageal varices. Although there are many causes of splenomegaly in tropical countries such as malaria and kala-azar we have found that $98 \%$ of our patients with upper GI bleeding who have palpable spleens were bleeding from varices. Further, associated lesions such as peptic ulcers and gastritis which have been found in up to $50 \%$ of American patients with portal hypertension are rare and not the source of bleeding in our patients.

If the spleen is not enlarged the patient is probably bleeding from an ulcer or erosive mucosal disease. A young man with no history of epigastric pain, who has bled only moderately, has a haemoglobin level of greater than $10 \mathrm{gmdl}^{-1}$ on admission and who has a recent history of taking drugs for fever or headaches, is 
likely to have erosive mucosal disease. A middle-age man with a history of dyspepsia lasting longer than a month, who has not taken analgesics and who has 3 a haemoglobin level of less than $10 \mathrm{gmdl}^{-1}$ on admission is more likely to be bleeding from an ulcer.

Step III: Low-risk and high-risk patients

There is a shortage of hospital beds in tropical countries and identifying patients who can be managed fairly adequately at home or in a primary health centre conserves these scarce hospital beds for those in the high-risk group who need them most.

Low-risk group. These are patients who are bleeding from gastritis and ulcers and not varices, who are below 40 years of age, who have had melaena without haematemesis, who have no dyspeptic symptoms for less than a month, who have a pulse rate of less than $100 \mathrm{~min}^{-1}$ and a systolic blood pressure of more than $100 \mathrm{mmHg}$. These patients can be managed at home with bed rest, ice-cold antacid sips every $2 \mathrm{~h}$ for 3 days and then put on a bland diet and mobilized. After 10 days the patient should be able to return to work. A barium meal or endoscopy should $\vec{c}$ then be performed if possible. If the patient has an ulcer he should be advised of appropriate drug treatment and if the test result is normal then the source of bleeding was probably erosive mucosal disease; he should be reassured and $\overbrace{\mathbb{D}}$ advised to avoid taking gastric irritants like chillies, aspirin and phenylbutazone $e_{\mathscr{O}}$

A few patients in the low-risk group will continue to bleed or will have $\frac{0}{9}$ recurrence of bleeding after it has been initially controlled by conservative measures. These patients should be moved into the high-risk group.

High-risk group. This includes all patients with variceal haemorrhage, those above 40 years of age, those who have had haematemesis, those who have had a history of dyspepsia of more than one month, those who have a pulse rate of above $100 \mathrm{~min}^{-1}$, a systolic blood pressure of less than $100 \mathrm{mmHg}$ and those who have been moved from the low-risk group. These patients should be treated in hospital.

\section{Step IV: Hospital treatment}

With the conventional resuscitation measures described earlier $60 \%$ of these patients will stop bleeding. The management of the remaining $40 \%$ will depend on the cause of bleeding. At this stage it is useful (but not essential) to perform a fibreoptic endoscopy. For:

(1) Variceal haemorrhage-the best treatment is endoscopic sclerotherapy but if not available, a Sengstaken-Blakemore double-ballooned tube should be inserted into the stomach and oesophagus and kept in position for $48 \mathrm{~h}$. Initially, only the gastric balloon should be inflated. Haemorrhage from varices can often be controlled with the gastric balloon alone when mild traction is put on the tube. This obviates some of the complications that can arise from inflating the oesophageal balloon particularly when nursing staff are not familiar with its use. The oesophageal 
balloon can be inflated subsequently if the gastric balloon is incapable of controlling the haemorrhage. If bleeding recurs surgery becomes necessary. The easiest procedure is to transfix the bleeding varix at the lower end of the oesphagus through a high gastrotomy using an abdominal approach. If expertise is available and the patient has only minimal ascites we advocate a splenectomy and a lieno renal shunt. This is effective in controlling the bleeding and is the definitive procedure for certain common causes of portal hypertension in tropical countries such as extrahepatic portal venous obstruction and non-cirrhotic portal fibrosis.

(2) Peptic ulcer and gastritis- The indications for operation are mainly a transfusion requirement of more than $1500 \mathrm{ml}$ in $24 \mathrm{~h}$ to maintain haemodynamic stability (and haemoglobin level) or a rebleed in hospital after initial control. Fibreoptic endoscopy, if available, may pinpoint the bleeding site and thereby make the operation easier.

The best operation for a bleeding gastric ulcer is a Billroth I partial gastrectomy unless the patient is very unstable or more than 60 years old when excision of the ulcer together with a truncal vagotomy and a drainage procedure should be done.

For a duodenal ulcer the best operation is to underrun the bleeding vessel through a $5 \mathrm{~cm}$ incision across the gastroduodenal junction and then do a truncal vagotomy and pyloroplasty. For ulcers larger than $2 \mathrm{~cm}$ or for post-bulbar ulcers we prefer a more radical procedure such as a vagotomy and antrectomy with a gastrojejunal anastomosis. With a smaller operation the incidence of rebleeding in the postoperative period is high.

(3) Erosive mucosal disease - A truncal vagotomy and antrectomy is probably the best procedure.

When the diagnosis has not been made preoperatively the surgeon should open the abdomen through an upper midline incision and then look for evidence of portal hypertension i.e. for an enlarged spleen which may not have been detected by abdominal palpation, for the characteristic pink appearance of the peritoneum and for distended tortuous veins in the omentum and over the bowel. If there is no portal hypertension the surface of the stomach and duodenum should be examined for ulcer scarring and then these structures should be palpated carefully by mobilizing the duodenum by a Kocher's manoeuvre and dividing the gastrocolic omentum to gain access to the posterior wall of the stomach. If no bleeding site is found the stomach is entered through an incision $5 \mathrm{~cm}$ long near the pylorus, the contained blood is evacuated and a lesion is sought, first by inserting a finger and carefully feeling the mucosa and then by visually inspecting the gastric and duodenal mucosae.

In very few cases no lesion is still found. The gall bladder and bile duct should be examined for the presence of blood, the liver should be thoroughly palpated (haemobilia) and the entire length of the pancreas visualized and palpated (haemosuccus pancreaticus).

There now remains the occasional patient in whom the cause of bleeding still cannot be found. We have encountered four such patients in all of whom we did a truncal vagotomy and antrectomy. In three the bleeding stopped. The fourth, a girl aged 13, rebled; we subsequently did a total gastrectomy but she again rebled and died. At post-mortem no cause of bleeding was found. 


\section{PROGNOSIS}

The effectiveness of any medical service dealing with patients who have had $\stackrel{\frac{3}{0}}{\frac{2}{2}}$ upper gastrointestinal bleeding is gauged not by the operative mortality alone $\overrightarrow{\vec{F}}$ (because if only good risk patients are operated upon the mortality will be low) $\frac{\mathcal{O}}{0}$ but by what proportion of patients who are admitted leave hospital alive. The mortality figures also depend on the mean age of the patients admitted and the predominant diagnosis. Thus mortality rates are higher when the patients are $\stackrel{\mathbb{Q}}{\Omega}$ older (Allan \& Dykes, 1976) as well as if the predominant cause of bleeding is oesophageal varices. (Dronfield et al. 1982)

The overall mortality of patients with upper GI bleeding ranges from $8-15 \%$ (Schiller et al., 1970; Hunt et al., 1979) and there has been little change in these figures ${ }_{\sigma}^{\omega}$ over the last 30 years. Occasionally there are reports of major improvements as a $\stackrel{\Phi}{\rightrightarrows}$ result of aggressive treatment in special medico-surgical units but these are few and far between. Newer techniques like electrocautery, (Wara, 1985) arteriography $\omega$ with selective vessel embolisation (Reuter, 1979) and laser photocoagulation (Steele, $\overrightarrow{8}$ 1989) have not made an appreciable impact on the mortality figures and are in any 음 case too expensive and sophisticated to use in tropical countries.

\section{OUR EXPERIENCE}

At the All India Institute of Medical Sciences between 1976 and 1989 we admitte 1480 patients to a combined medico-surgical unit dealing with upper GI haemo rhage. The mean age of our patients was 43 years and males predominated over females in a ratio of $3 \cdot 2: 1$. The causes of bleeding in our patients are shown in Figure 1. In 1080 patients $(73 \%)$ bleeding was controlled by the initial conservative management. However, of these 162 (15\% of the 1080 patients) died without operation either because they rebled in hospital and this rebleeding was so massive that they could not be taken to the operating theatre or because they were in deep coma from liver failure and operation was not advised. A total of 400 (27\%) patients underwent an operation of which 88 (22\% of the operated patients) died (i.e. $6 \%$ of all patients presenting initially with bleeding [Table 2]).

Thus $1230(83 \%)$ of the 1480 patients admitted left hospital alive. This mortality rate is high because although our patients are young a large proportion have portal

Table 2. Results of upper gastrointestinal haemorrhage at All India Institute of Medical Sciences $(n=1480)$

\begin{tabular}{lrr}
\hline Management & $n$ & Mortality \\
\hline Conservative & 1080 & $162(15 \%)$ \\
Operation & 400 & $88(22 \%)$ \\
Total & 1480 & $250(17 \%)$ \\
\hline
\end{tabular}




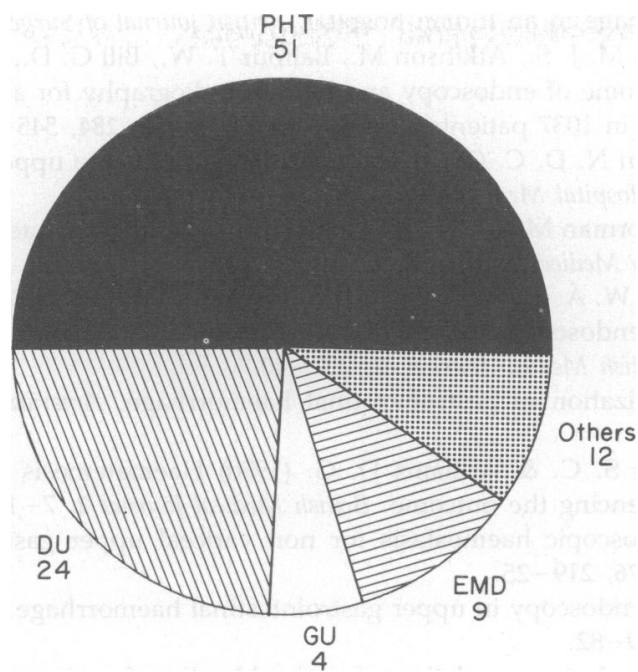

Fig. 1. The causes of upper gastrointestinal bleeding in 1480 patients at the All India Institute of Medical Sciences (1976-1989). PHT-portal hypertension; DU-duodenal ulcer; GU-gastric ulcer; EMDerosive mucosal disease.

hypertension. Also sometimes there is difficulty in getting blood for transfusions. This delays an urgent operation and may have been a factor in raising the mortality figures.

\section{CONCLUSION}

Upper gastrointestinal haemorrhage is a common emergency in tropical countries. In these countries the patients with upper GI haemorrhage are usually young, poor, male and the source of bleeding in half of them is oesophageal varices. They can be fairly well managed using a stepwise approach consisting of resuscitation, diagnosis, division into a low and a high risk group and hospital treatment. Endoscopy facilities although desirable are not essential.

The mortality of patients with major bleeding admitted to the All India Institute of Medical Sciences, New Delhi, India between 1976 and 1989 was 17\%.

\section{REFERENCES}

Allan R. \& Dykes P. (1976) Study of factors influencing mortality rates from upper gastrointestinal haemorrhage. Quarterly Journal of Medicine, 115, 533-50.

Anand C. S., Tandon B. N. \& Nundy S. (1983) The causes, management and outcome of upper 
gastrointestinal haemorrhage in an Indian hospital. British Journal of Surgery 70, 209-11.

Dronfield M. W., Langman M. J. S., Atkinson M., Balfour T. W., Bill G. D., Vella K. D., Amar S. S. \& Knapp D. R. (1982) Outcome of endoscopy and barium radiography for acute upper gastrointestinal bleeding: controlled trial in 1037 patients. British Medical Journal, 284, 545-8.

Forrest J. A. H. \& Finlayson N. D. C. (1974) The investigation of acute upper gastrointestinal haemorrhage. British Journal of Hospital Medicine, Aug 24, 160-5.

Hunt P. S., Hansky J. \& Korman M. G. (1989) Mortality in patients with haematemesis and melaena: a prospective study. British Medical Journal, 1, 1238-40.

Morgan A. G. H., McAdam W. A. F., Walmsley G. L., Jessop A., Horrocks J. C. \& de Dombal F. T. (1977) Clinical findings, early endoscopy and multivariate analysis in patients bleeding from the upper gastrointestinal tract. British Medical Journal, 2, 237-40.

Reuter S. R. (1979) Embolization of gastrointestinal haemorrhage. American Journal of Roentgenology, 133, 557-8.

Schiller K. F. R., Truelove S. C. \& Williams D. G. (1970) Haematemesis and melaena with special reference to factors influencing the outcome. British Medical Journal 2, 7-14.

Steele R. J. C. (1979) Endoscopic haemostasis for non variceal upper gastrointestinal haemorrhage. British Journal of Surgery 76, 219-25.

Tandon R. K. (1978) Early endoscopy in upper gastrointestinal haemorrhage. Journal of the Association of Physicians of India, 26, 279-82.

Wara P. (1985) Endoscopic electrocoagulation of major bleeding from peptic ulcer. Acta Chiropractica Scandinavica, 151, 29-35.

Wicks A. C. B., Thomas G. E. \& Clair D. J. (1975) Comparison of fibreoptic endoscopy in acute upper gastrointestinal haemorrhage in Africans and Europeans. British Medical Journal, 4, 259-60.

Yajko R. D., Norton L. W. \& Eiseman B. (1975) Current management of upper gastrointestinal bleeding. Annals of Surgery, 181, 474-80. 